\title{
Modeling of the Surface Impedance of Conductive Ferromagnetic Films on Different Substrates
}

\author{
T. NurgalieV \\ Institute of Electronics, Bulgarian Academy of Sciences \\ 72 Tsarigradsko Chausee, 1784 Sofia, Bulgaria
}

The microwave surface impedance of conductive ferromagnetic films hypothetically deposited on dielectric, metal and superconducting substrates was numerically investigated for the case when the film is tangentially magnetized. It was shown that the surface impedance is governed by the ferromagnetic film conductivity if the film is thin enough. Peculiarities of the impedance caused by the ferromagnetic resonance arise more clearly in thicker ferromagnetic films or in films deposited on substrates of higher permittivity or on metal and superconducting substrates. A ferromagnetic film strongly affects the surface impedance of superconducting material, especially, in the ferromagnetic resonance frequency region.

PACS numbers: 75.47.Lx, 75.70.-i

\section{Introduction}

The interest to the conductive ferromagnetic (FM) manganites (for example, $\mathrm{La}_{0.7} \mathrm{Sr}_{0.3} \mathrm{MnO}_{3}$ ) is caused by their original physical characteristics [1]. Thin films of the manganites can be grown on the dielectric (for example, $\mathrm{LaAlO}_{3}$ and $\mathrm{SrTiO}_{3}$ ) and high temperature superconducting (HTS) (for example, $\mathrm{YBa}_{2} \mathrm{Cu}_{3} \mathrm{O}_{7}$ ) materials. The manganites are suitable for the preparation of HTS/FM heterostructures which are prospective for application in spintronics [2-4]. The heterostructures of such kind can demonstrate negative refraction at millimeter waves and can be used as metamaterials as well [5].

The ferromagnetic resonance (FMR) [6] and magneto-impedance effects can be observed in the manganites at microwave frequencies. Conventionally, the EPR spectrometry is used for investigation of microwave characteristics of thin films of FM manganites [7]. In this paper the microwave surface impedance of the conductive FM films deposited on the dielectric, metal, and the HTS materials were investigated through the modeling. The results allow to conclude that the impedance measurement can be succesfully used for investigation of the FM film parameters in the HTS/FM layered structures. 


\section{Modeling procedure and parameters}

Let us consider a layered structure consisting of a ferromagnetic manganite thin film deposited on dielectric, metal, or HTS substrates. External DC magnetic field $\boldsymbol{H}$ is applied parallel to the surface of the FM film and the linearly polarized planar transverse electromagnetic wave (microwave magnetic $\boldsymbol{h}$ field of which is parallel to the film surface and perpendicular to the external field direction) impinges normally on the layered structure. The time-harmonic Maxwell, Landau-Lifshitz and London equations govern the electromagnetic waves behaviors in the structures containing the dielectric, ferromagnetic, and superconducting (SC) components. The solutions of these equations for the determination of the surface impedance of a layered structure (which can be obtained in the form equivalent to the formulas of the impedance transformation rule as well) were used for modeling the surface impedance of the conductive FM film deposited on different substrates.

The modeling parameters were the following: the thickness of the dielectric substrates is $0.5 \mathrm{~mm}$ and the permittivity of the substrates is $\varepsilon_{d}=24-0.00001 \mathrm{i}$ and $\varepsilon_{d}=600-15 \mathrm{i}$ (the substrates of type of $\mathrm{LaAlO}_{3}$ and $\mathrm{SrTiO}_{3}$ ); the resistivity of the metal substrate is $\rho_{\mathrm{m}}=4.3 \times 10^{-9} \Omega \mathrm{m}$ (good conductive metals as Ag and $\mathrm{Cu}$ at $77 \mathrm{~K}$ ); the surface resistance $R_{\mathrm{S}}($ at $8 \mathrm{GHz})$ and the London penetration depth $\lambda_{\mathrm{L}}$ of the superconductor substrate are $R_{\mathrm{S}}=1 \mathrm{~m} \Omega$ and $\lambda_{\mathrm{L}}=0.3 \mu \mathrm{m}$ (which corresponds to the parameters of the high temperature superconducting $\mathrm{YBa}_{2} \mathrm{Cu}_{3} \mathrm{O}_{7}$ material at $77 \mathrm{~K}$ ); the resistivity $\rho_{\mathrm{FM}}$, the saturation magnetization $M$ and the Landau-Lifshitz damping parameter $a$ for the FM film were assumed to be $\rho_{\mathrm{FM}}=5.1 \times 10^{-6} \Omega \mathrm{m}, \mu_{0} M=714 \mathrm{mT}$ [8], $\alpha=0.01$ (which are similar to the parameters of $\mathrm{La}_{0.7} \mathrm{Sr}_{0.3} \mathrm{MnO}_{3}$ materials at $77 \mathrm{~K}$, although in the thin films the value of $M$ can be smaller up to several times [1]); $H=60 \mathrm{mT}$.

\section{Results and discussion}

Results of modeling of the real part of the effective microwave surface impedance $Z_{\mathrm{ef}}$ of the conductive FM films of different thicknesses deposited on dielectric substrates of two different permittivites are shown in Fig. 1.

Frequency dependence of the impedance of the clean (without the FM film) substrates (Fig. 1a and b, curves 1) are due to the finite thickness of these substrates. A very thin FM film deposited on the substrates of low permittivity is characterized nearly by the same surface impedance $\operatorname{Re}\left(Z_{\mathrm{ef}}\right)$ as a clean substrate (Fig. 1a, curves 2). A decrease in the impedance with the increase in the FM film thickness is due mainly to the conductivity of the film if the film is thin enough (Fig. 1a, curves 2-4). The ferromagnetic resonance (FMR) affects the impedance of the thicker films deposited on the substrates of low permittivity (Fig. 1a, the peaks in the dependences $5-7$ ).

The peculiarities of the impedance caused by FMR can be clearly observed in very thin FM films if they are deposited on the substrates of higher permit- 

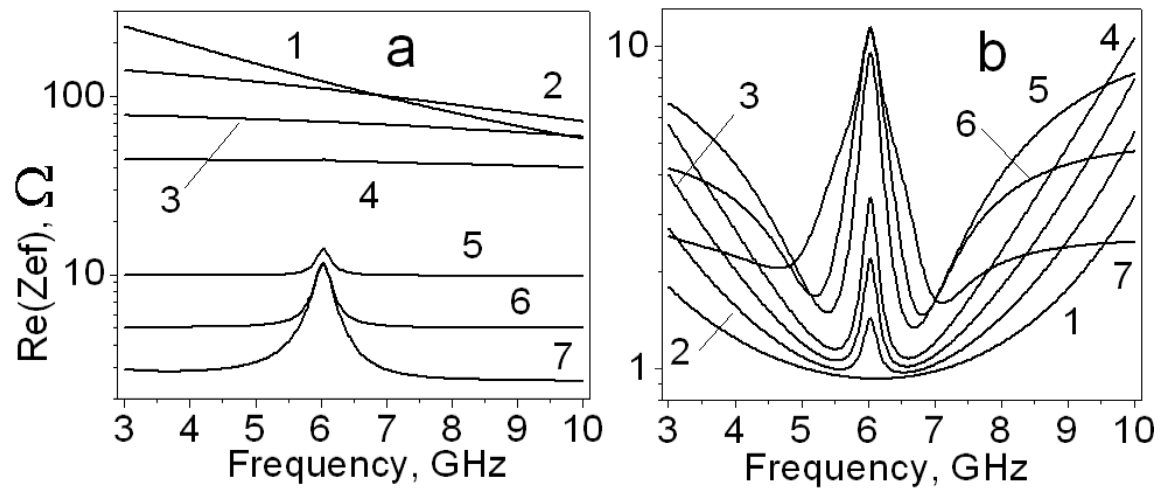

Fig. 1. Frequency dependence of the real part of the effective surface impedance $\operatorname{Re}\left(Z_{\mathrm{ef}}\right)$ of the manganite films deposited on the dielectric substrates (with the thickness of $0.5 \mathrm{~mm}$ ) with the permittivity $\varepsilon_{d}=24-0.00001 \mathrm{i}$ (a) and $\varepsilon_{d}=600-15 \mathrm{i}$ (b). The thickness of the manganite film is $0,0.02,0.05,0.1,0.5,1,2 \mu \mathrm{m}$ (curves $1-7$, respectively).
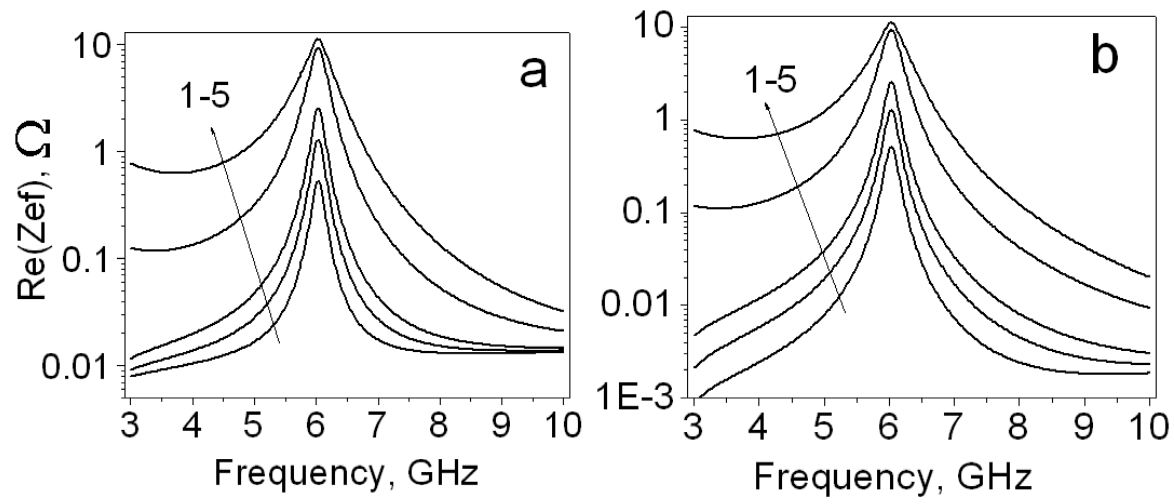

Fig. 2. Frequency dependence of the real part of the effective surface impedance $\operatorname{Re}\left(Z_{\mathrm{ef}}\right)$ of the manganite films deposited on the metal (a) and superconductor (b) substrates. The thickness of the manganite film is $0.02,0.05,0.1,0.5,1 \mu \mathrm{m}$ (curves $1-5$, respectively).

tivity (Fig. 1b, a peak in the dependence 2). On the other hand, the possible standing wave resonances excited acrosss the substrate of high permittivity can strongly affect the impedance of thin FM film leading to its significant frequency dependence.

It can be noted that the real part of $Z_{\text {ef }}$ for the very thin (which are nearly transparent) conductive films deposited on dielectric substrates does not reflect exactly the absorbed microwave power, but the power which enters the film. A part of this power can pass through the film-substrate system and propagate forward. The situation is different for the cases of the metal and superconductor substrates and the real part of the effective surface impedance $\operatorname{Re}\left(Z_{\mathrm{ef}}\right)$ characterizes the microwave losses in the FM film/substrate system in this case. 
Results of modeling of $\operatorname{Re}\left(Z_{\text {ef }}\right)$ of the conductive FM films deposited on the good conductive metal and the superconductive substrates are shown in Fig. 2. As the conductivity of the FM film is lower than that of the substrates, the real part of the impedance increases with increase in the film thickness. Contribution of the FMR to $\operatorname{Re}\left(Z_{\text {ef }}\right)$ is great enough in even very thin FM films when the film is deposited on the conductive substrates (Fig. 2, curves 1). The highest sensitivity of the impedance $Z_{\text {ef }}$ to the FM film parameters is observed if a thin FM film is deposited on a SC substrate (Fig. 2b). In principle, this allows to obtain information about the FM films in the FM/SC (for example, $\mathrm{La}_{0.7} \mathrm{Sr}_{0.3} \mathrm{MnO}_{3} / \mathrm{YBa}_{2} \mathrm{Cu}_{3} \mathrm{O}_{7}$ ) layered structures by measuring of $Z_{\text {ef }}$ of the structure. For this reason the parallel plate resonator technique was considered recently [9] as one of the candidates for the such kind of investigations.

\section{Conclusions}

The microwave surface impedance of conductive FM films hypothetically deposited on the dielectric, metal, and SC substrates was numerically investigated. The highest sensitivity of the impedance $Z_{\text {ef }}$ to the FM film parameters is observed if a thin FM film is deposited on a SC material. The results allow to conclude that the impedance measurement can be succesfully used for investigation of the FM film parameters in the HTS/FM structures.

\section{Acknowledgments}

This work was partially supported by the Bulgarian Science Fund under Contract F 1503/05.

\section{References}

[1] A.-M. Haghiri-Gosnet, J.-P. Renard, J. Phys. D, Appl. Phys. 36, R123 (2003).

[2] S. Soltan, J. Albrecht, H.-U. Habermeier, Phys. Rev. B. 70, 144517 (2004).

[3] V. Pena, Z. Sefrioui, C. Leon, J. Santamaria, M. Varela, S.J. Pennycook, J.L. Martinez, Phys. Rev. B 69, 224502 (2004).

[4] P. Przyslupski, I. Komissarov, W. Paszkowicz, P. Dluzewski, R. Minikayev, M. Sawicki, Phys. Rev. B 69, 134428 (2004).

[5] A. Pimenov, A. Loidl, P. Przyslupski, B. Dabrowski, Phys. Rev. B 95, 247009 (2006).

[6] A. Schwartz, M. Scheffler, S. Anlage, Phys. Rev. B 61, R870 (2000).

[7] D.L. Lyfar, S.M. Ryachenko, V.N. Krivoruchko, S.I. Khartsev, A.M. Grishin, Phys. Rev. B 69, 100409 (2004).

[8] K. Steenbeck, R. Hiergeist, Appl. Phys. Lett. 75, 1 (1999).

[9] T. Nurgaliev, B. Blagoev, T. Donchev, S. Miteva, P.B. Mozhaev, J.E. Mozhaeva, G.A. Ovsyannikov, I.M. Kotelyanskii, C. Jacobsen, J. Phys. Conf. Series 43, 329 (2006). 